\title{
Torque teno sus virus 1 and 2 viral loads in faeces of porcine circovirus 2-positive pigs
}

\author{
Alessandra M. M. G. de Castro ${ }^{1,3}$, Cintia M. Baldin ${ }^{1}$, Cintia M. Favero ${ }^{1}$, \\ Priscilla F. Gerber ${ }^{2}$, Rafael I. Cipullo ${ }^{1}$, Leonardo J. Richtzenhain ${ }^{1}$ \\ ${ }^{1}$ University of São Paulo, College of Veterinary Medicine, Department of Preventive Veterinary Medicine \\ and Animal Health, São Paulo, Brazil \\ ${ }^{2}$ Federal University of Minas Gerais, Department of Pathology, Minas Gerais, Brazil \\ ${ }^{3}$ Complexo Educacional Faculdade Metropolitanas Unidas, Faculdade de Medicina Veterinária, São Paulo, Brazil
}

Received February 10, 2014

Accepted November 26, 2014

\begin{abstract}
Recently, studies have suggested an association between the Torque teno sus virus (TTSuV) and the Porcine circovirus-2 (PCV2) in PCV2-associated disease cases. The aim of this study was to verify TTSuVs loads in pig faeces from PCV2-positive animals with and without diarrhea from PCVADaffected and PCV2-unvaccinated herds. A total of 80 faecal samples were collected individually from nursery and grow-finish pigs with $(n=40)$ or without $(n=40)$ diarrhea. The samples were tested for PCV2 and TTSuVs by using DNA binding dye SYBR Green quantitative polymerase chain reaction (qPCR). Torque teno sus virus $k 2$ (TTSuVk2) load in the faeces was significantly higher in the nursery pigs with diarrhea, and these pigs also exhibited significantly higher PCV2 $(P<0.01)$ faecal matter loads compared to the non-diarrheic animals from the same age group. Torque teno sus virus 1 (TTSuV1) viral loads were the same regardless of age group and disease condition. There were no correlations between PCV2 and TTSuV1 or TTSuVk2 and TTSuV1 viral loads; however, a weak correlation $(r=0.23, P=0.03)$ was found between TTSuVk2 and PCV2 viral loads. In conclusion, TTSuVk2 viral loads were significantly higher in the diarrheic faeces from the nursery pigs. Additionally, the higher loads of PCV2 and TTSuVk2 in the nursery-diarrheic animals revealed that diarrhea might have an important role in the spread of both viruses in herds.
\end{abstract}

Epidemiology, swine virus, diarrhea, disease

Torque teno sus virus (TTSuV) is classified within the Anelloviridae family and Iotatorquevirus genus and comprises the following species: Torque teno sus virus $1 a$ (TTSuV1a) and Torque teno sus virus 1b (TTSuV1b) (ICTV 2013). The genus Kapatorquevirus comprises only one species, Torque teno sus virus $k 2$ (TTSuVk2). The TTSuV1 and TSuVk2 are distributed in several countries with frequencies ranging from $24 \%$ to $100 \%$ (Kekarainen et al. 2006; Taira et al. 2009). The pathogenic role of TTSuV is not clear because this virus infects a relatively high proportion of animals that are apparently healthy (Segalés et al. 2009). The TTSuV has been considered nonpathogenic; however, studies have shown that it can serve as a 'trigger' or cofactor for Porcine circovirus 2 (PCV2) in the porcine circovirus 2-associated disease (PCVAD) (Krakowka et al. 2000). This association between TTSuVs and PCV2 infection has been suggested because TTSuVk2 prevalence is significantly higher in post-weaning multi-systemic wasting syndrome (PMWS) affected animals than in healthy domestic swine (Kekarainen et al. 2006). However, no differences between TTSuV loads in PCVAD-affected and non-affected pigs were found in another study (Lee et al. 2010). Coinfections with PCV2 and TTSuVs in reproductive organs, semen and ovarian follicular fluid exhibit no correlation between TTSuVs and reproductive failure (Ritterbusch et al. 2012). Additionally, in a double-blinded, randomized trial included in a PCV2 vaccination study, the vaccination of PCV2 sub-clinically infected pigs did not modify the outcome

Address for correspondence:

Dr. Alessandra M.M.G. de Castro

Complexo Educacional Faculdade Metropolitanas Unidas

Faculdade de Medicina Veterinária

Rua Ministro Nélson Hungria, 541

Real Parque, São Paulo, SP, Brazil

Phone: +55 11 30917704; 24

E-mail: alessandramarnie@gmail.com

http://actavet.vfu.cz/ 
of TTSuVk2 infection (Nieto et al. 2012). The role of TTSuVs co-infections in PCV2 faecal shedding is unknown, and even less is known about this role in PCV2-unvaccinated PCV2-positive swine. The aim of this study was to verify TTSuVs loads in pig faeces with and without diarrhea from PCV2-positive animals from PCVAD-affected and PCV2unvaccinated herds.

\section{Materials and Methods}

A total of 80 faecal samples were individually collected without environmental contamination from pigs from two PCVAD-affected and PCV2-unvaccinated herds in the State of São Paulo, Brazil between July 2008 and July 2009. PCVAD was defined as a significant increase in post-weaning mortality and 'wasting' rates and by the detection of PCV2 nucleic acid by polymerase chain reaction (PCR) in at least $50 \%$ of the pigs that died with clinical signs of PCVAD. The wasting rates were determined as the percentages of animals that were delayed in or never reached slaughter weight and were discarded prior to slaughter, relative to the total numbers of animals that were slaughtered during the year-long period. None of herds had been subjected to PCV2-vaccination protocols at the time of the study. In each herd, the same numbers of nursery ( $\pm 35-70$ days) and grow-finish $( \pm 35-150$ days) pigs were randomly selected based on the presence $(n=20)$ or absence of diarrhea $(n=20)$ at the moment of sample collection. The faeces were collected by massaging the pig's abdomen and placed in a sterile bottle that was conditioned in ice during transport to the laboratory. In the laboratory, the samples were homogenized in $20 \%$ TE buffer $(10 \mathrm{mM}$ Tris- $\mathrm{HCl}, 1 \mathrm{mM}$ EDTA, $\mathrm{pH} 8.0)$ and stored at $-20{ }^{\circ} \mathrm{C}$ until further processing. DNA was extracted from the faecal suspensions using a phenol-chloroform and proteinase $\mathrm{K}$ protocol that was previously described by Sambrook et al. (1989). The extracts were subsequently used for the detection and quantification of PCV2, TTSuV1, and TTSuVk2 using primers that were previously described by Yang et al. (2007) and Segalés et al. (2009). Quantitative PCR was performed using SYBR green chemistry and a StepOne Real-Time PCR system (Applied Biosystems, Canada) under universal conditions. The numbers of copies of viral DNA were determined by comparisons with a standard curve, and the viral concentrations are expressed as the $\log _{10}$ PCV2 and TTSuVs DNA per $100 \mathrm{ng}$ of total DNA in the faecal samples. The extracted samples were also tested for $\beta$-actin with PCRs as a control as previously described by Hui et al. (2004) to detect possible PCR false-negative results due to failures in DNA extraction or the presence of PCR inhibitors. All samples were positive for $\beta$-actin.

Shapiro-Wilk's test was used to evaluate the normalities of data distributions of the examined variables. Nonparametric Mann-Whitney test was used to analyze PCV2, TTSuV1, and TTSuVk2 viral loads within each age group between the diseased and healthy animals. Spearman's correlations were used to examine the relationships between the viral loads of PCV2, TTSuV1, and TTSuVk2 in the animals. The results were considered significant at $P<0.05$.

\section{Results}

In the present study, the TTSuVk2 load was significantly higher in the faeces of the pigs with diarrhea in the nursery age-group $(P<0.01)$, and these animals also exhibited significantly higher PCV2 loads $(P<0.01)$ in the faecal samples compared to the nondiarrheic animals from the same age group (Table 1). The PCV2 loads in faeces of the

Table 1. Prevalence (number of animals positive/total number per group) and the means \pm the standard deviations of the $\log _{10}$ viral copy numbers per $100 \mathrm{ng}$ of fecal total DNA for the pigs with and without diarrhea in nursery and growfinish age groups.

\begin{tabular}{lcccr}
\hline \multicolumn{5}{c}{ Number of positive animals (viral load) } \\
\hline Age group & Diarrhea & PCV2 & TTSuV1 & TTSuVk2 \\
\hline \multirow{2}{*}{ Nursery } & No & $20 / 20(3.49 \pm 1.05)^{\mathrm{a}}$ & $12 / 20(2.19 \pm 1.96)$ & $3 / 20(0.57 \pm 1.39)^{\mathrm{a}}$ \\
& Yes & $20 / 20(4.90 \pm 1.49)^{\mathrm{b}}$ & $10 / 20(1.69 \pm 1.80)$ & $12 / 20(2.21 \pm 1.91)^{\mathrm{b}}$ \\
\hline \multirow{2}{*}{ Grow-finish } & No & $20 / 20(4.38 \pm 1.25)$ & $8 / 20(1.32 \pm 1.72)$ & $13 / 20(2.64 \pm 2.16)$ \\
& Yes & $20 / 20(4.00 \pm 1.38)$ & $10 / 20(1.63 \pm 1.75)$ & $8 / 20(1.65 \pm 2.16)$ \\
\hline
\end{tabular}

Different superscripts within the column indicate significant differences $(P<0.05$, Mann-Whitney test $)$ between groups. PCV2 - Porcine circovirus 2

TTSuV1 - Torque teno sus virus 1

TTSuVk2 - Torque teno sus virus $k 2$ 
pigs without diarrhea ranged from $10^{2}$ to $10^{4}$, and $10^{2}$ to $10^{6} \mathrm{PCV} 2 \mathrm{DNA}$ copies/100 ng of total DNA in the nursery and grow-finish pigs, respectively. Among the diarrheic animals, the PCV2 loads in faecal samples ranged from $10^{2}$ to $10^{7}$, and $10^{3}$ to $10^{8}$ PCV2 DNA copies/100 ng of total DNA for the nursery and grow-finish pigs, respectively. Concurrent TTSuV1 and PCV2 infections were detected in 55\% (22/40) and 45\% (18/40) of faecal samples from the nursery and grow-finish pigs, respectively. Concurrent TTSuVk 2 and PCV2 infections were present at a similar rate; i.e., these infections were present in $37.5 \%$ $(15 / 40)$ and $52.5 \%(21 / 40)$ of the nursery and grow-finish pigs, respectively. However, when only nursery pigs were considered, the rate of concurrent TTSuVk2 and PCV2 infections was higher in diarrheic faecal samples (12/20) than in non-diarrheic samples (3/20). In contrast, concurrent TTSuV1 and PCV2 infections were found in similar rates across the age groups. There were no correlations between the viral loads of PCV2 and TTSuV1 or those of TTSuVk2 and TTSuV1. Weak correlation $(r=0.2367, P=0.03)$ was found between TTSuVk2 and PCV2 viral loads. Regarding triple co-infections, 17.5\% $(7 / 40)$ and $30 \%(12 / 40)$ of the diarrheic and non-diarrheic faecal samples, respectively, exhibited such infections, and the rates of triple infections were $20 \%(4 / 20)$ and $40 \%(8 / 20)$ in the nursery and grow-finish pigs, respectively. There were no differences between the groups in the occurrence of triple co-infection in the faecal samples.

\section{Discussion}

The effects of TTSuVs and PCV2 co-infection in swine herds remain controversial. The introduction of the worldwide use of PCV2 vaccination has made this type of study (i.e., studies of the interaction between of these two agents in fields) even more difficult because PCV2 vaccine can interfere with the data. Thus, TTSuVs loads in the faeces of pigs with and without diarrhea from PCV2-positive animals from PCVAD-affected and PCV2-unvaccinated herds were evaluated. The faecal TTSuVk2 load was significantly higher in pigs with diarrhea in the nursery age group $(P<0.01)$; this group also exhibited a significantly higher faecal PCV2 load $(P<0.01)$ compared to the non-diarrheic animals from the same age group (Table 1). This higher faecal TTSuVk2 load exhibited a pattern similar to that which was previously observed for TTSuVs in sera; i.e., higher serum TTSuVk2 but not TTSuV1 viral loads was observed in the sera of PMWS-affected pigs (Aramouni et al. 2010). The pathogenic role of TTSuV has not yet been demonstrated, and its role during co-infection with other pathogens is under debate (Kekarainen and Segalés 2009). In contrast to TTSuVk2, TTSuV1 load ranged from $10^{2}$ to $10^{4}$ copies of viral DNA/100 ng of total faeces regardless of the clinical status and age group. This result is in accordance with those of other studies in which viraemia was evaluated and no differences were observed between healthy and diseased animals (Aramouni et al. 2010; $\mathrm{Nieto}$ et al. 2012). Interestingly, there were no differences between the mean viral loads of any of the tested viruses between the pigs with and without diarrhea in the grow-finish age group. The TTSuVk2 load was slightly increased in the faeces of animals without diarrhea $\left(10^{3}\right.$ to $10^{4}$, and $10^{2}$ to $10^{6}$ for the nursery and grow-finish pigs, respectively), and similar loads were observed in animals with diarrhea regardless of age $\left(10^{2}\right.$ to $\left.10^{5}\right)$. Notably, TTSuV1 and TTSuVk2 loads in healthy pigs, and TTSuV1 loads in PCVAD-affected animals increase until 11 weeks of age and decline thereafter, and TTSuVk2 DNA loads in PCVAD-affected pigs increase until 15 weeks of age (Nieto et al. 2012). In tissues, the mean amounts of TTSuVs DNA increase with age (Aramouni et al. 2010). As has been observed in PCV2 infections (Opriessnig et al. 2007), this behavior might be indicative of inefficient immune responses to the virus. All samples were positive for PCV2. High rates of PCV2-positive faecal samples were previously reported in experimentally and naturally infected animals (Opriessnig and Halbur 2012). Notably, diarrhea is not a 
suitable clinical sign of PCV2 infection. However, it should be noted that all of the samples were PCV2-positive and that PCV2 or TTSuVk2 viral loads were higher in the nurserydiarrheic animals, which indicates that diarrhea might have an important role in the spread of both viruses in herds, particularly among this age group. Currently, little information about TTSuVs shedding in faeces is available, and even less information is available about PCV2-unvaccinated, PCVAD-positive herds because vaccination against PCV2 has been a common practice adopted by pig producers worldwide. Therefore, given the low number of samples tested herein, we did not aim to represent the country; rather we sought to examine non-PCV2-immunized animals. The PCV2 vaccination decreases the viral loads in tissue, faeces, and nasal secretions and also decreased viraemia in naturally and experimentally infected swine (Chae 2012). Furthermore, it is known that PCV2 infection affects functionality of the pigs' immune systems due to lymphoid depletion which is triggered by combination of several mechanisms including apoptosis, viral-induced lysis, and destruction of lymphoid structures (Darwich and Mateu 2012). Therefore, PCV2 infection without interference from PCV2 vaccination might have facilitated the replication of TTSuVs; similar phenomena have been observed for other agents. Singular PCV2 infection rarely results in clinical disease; however, PCVAD is often accelerated in onset, enhanced in severity and prolonged in duration by concurrent viral or bacterial infections. The PCV2 has also been shown to enhance protozoal, metazoal, and fungal infections as observed in retrospective and cross-sectional studies of the presence and prevalence of various infectious agents associated with PCVAD in field conditions (Opriessnig and Halbur 2012). The requirement of other co-agents of the development of PCVAD is wellknown, and the results of field studies are essential to guide experimental studies. To the authors' knowledge, this is the first study in which PCV2 and TTSuVs DNA loads were quantified in the faeces of naturally co-infected animals before the introduction of PCV2 vaccination. In conclusion, higher loads of PCV2 and TTSuVk2 in the nursery-diarrheic animals indicate that diarrhea might have an important role in the spread of both viruses across herds.

\section{Acknowledgments}

The authors would like to thank FAPESP for funding this study (grant no. 2007/57115-3). The CMB received a scholarship from FAPESP (grant no. 08/58822-8). The authors would like to thank the swine farms that provided samples for this study.

\section{References}

Aramouni M, Segalés J, Cortey M, Kekarainen T 2010: Age-related tissue distribution of swine Torque teno sus virus 1 and 2. Vet Microbiol 146: 350-353

Chae C 2012: Commercial porcine circovirus type 2 vaccines: efficacy and clinical application. Vet J 194: 151157

Darwich L, Mateu E 2012: Immunology of porcine circovirus type 2 (PCV2). Virus Res 164: 61-67

Hui RKH, Zeng F, Chan CMN, Yuen KY, Peiris JSM, Leung FCC 2004: Reverse transcriptase PCR diagnostic assay for the coronavirus associated with severe acute respiratory syndrome. J Clin Microbiol 42: 1994-1999

ICTV (International Committee on Taxonomy of Viruses Virus Taxonomy) 2013: Release (current). Available at: http://ictvonline.org/virusTaxonomy.asp?version=2013. Accessed August 15, 2014

Kekarainen T, Segalés J 2009: Torque teno virus infection in the pig and its potential role as a model of human infection. Vet J 180: 163-168

Kekarainen T, Sibila M, Segalés J 2006: Prevalence of swine Torque teno virus in post-weaning multisystemic wasting syndrome (PMWS)-affected and non-PMWS-affected pigs in Spain. J Gen Virol 87: 833-837

Krakowka S, Ellis JA, Meehan B, Kennedy S, McNeilly F, Allan G 2000: Viral wasting syndrome of swine: experimental reproduction of postweaning multisystemic wasting syndrome in gnotobiotic swine by coinfection with porcine circovirus 2 and porcine parvovirus. Vet Pathol 37: 254-263

Lee SS, Sunyoung S, Jung H, Shin J, Lyoo YS 2010: Quantitative detection of porcine torque teno virus in porcine circovirus-2-negative and porcine circovirus-associated disease affected pigs. J Vet Diagn Invest 22: 261-264 
Nieto D, Aramouni M, Sibila M, Fraile L, Kekarainen T, Segalés J 2012: Lack of effect of piglet vaccination against Porcine circovirus type 2 (PCV2) on serum viral loads of Torque teno sus virus 2 (TTSuV2). Vet Microbiol 157: 8-12

Opriessnig T, Halbur PG 2012: Concurrent infections are important for expression of porcine circovirus associated disease. Virus Res 164: 20-32

Opriessnig T, Meng XJ, Halbur PG 2007: Porcine circovirus type 2 associated disease: update on current terminology, clinical manifestations, pathogenesis, diagnosis, and intervention strategies. J Vet Diagn Invest 19: $591-615$

Ritterbusch GA, Rocha CA, Mores N, Simon NL, Zanella EL, Coldebella A, Ciacci-Zanella JR 2012: Natural co-infection of torque teno virus and porcine circovirus 2 in the reproductive apparatus of swine. Res Vet Sci 92: $519-523$

Sambrook J, Fitsch EF, Maniatis T 1989: Molecular Cloning. In: Sambrook J, Fitsch EF, Maniatis T (Eds): A Laboratory Manual. Cold Spring Harbor, New York, pp. 65-69

Segalés J, Martínez-Guinó L, Cortey M, Navarro N, Huerta E, Sibila M, Pujols J, Kekarainen T 2009: Retrospective study on swine Torque teno virus genogroups 1 and 2 infection from 1985 to 2005 in Spain. Vet Microbiol 134: 199-207

Taira O, Ogawa H, Nagao A, Tuchiya K, Nunoya T, Ueda S 2009: Prevalence of swine Torque teno virus genogroups 1 and 2 in Japanese swine with suspected post-weaning multisystemic wasting syndrome and porcine respiratory disease complex. Vet Microbiol 139: 347-350

Yang ZZ, Habib M, Shuai JB, Fang WH 2007: Detection of PCV2 DNA by SYBR Green I based quantitative PCR. J Zhejiang Univ - Sci B 8: 162-169 